Published on Web 09/10/2002

\title{
Molecular Rectification in a Metal-Insulator-Metal Junction Based on Self-Assembled Monolayers
}

\author{
Michael L. Chabinyc, ${ }^{\dagger}$ Xiaoxi Chen, ${ }^{\dagger}$ R. Erik Holmlin, ${ }^{\dagger}$ Heiko Jacobs, ${ }^{\dagger}$ \\ Hjalti Skulason,‡ C. Daniel Frisbie,‡ Vladimiro Mujica,§ Mark A. Ratner,§ \\ Maria Anita Rampi, ${ }^{*}, \|$ and George M. Whitesides*,† \\ Contribution from the Department of Chemistry and Chemical Biology, Harvard University, \\ 12 Oxford Street, Cambridge, Massachusetts 02138, Dipartimento di Chimica, Centro di \\ Fotochimica CNR, Universita' di Ferrara, 44100 Ferrara, Italy, Department of Chemical \\ Engineering and Materials Science, University of Minnesota, 421 Washington Avenue SE, \\ Minneapolis, Minnesota 55455, and Department of Chemistry, Northwestern University,
} Evanston, Illinois 60208

Received April 8, 2002

\begin{abstract}
An electrical junction formed by mechanical contact between two self-assembled monolayers (SAMs)-a SAM formed from an dialkyl disulfide with a covalently linked tetracyanoquinodimethane group that is supported by silver (or gold) and a SAM formed from an alkanethiolate SAM that is supported by mercury-rectifies current. The precursor to the SAM on silver (or gold) was bis(20-(2-((2,5-cyclohexadiene1,4-diylidene)dimalonitrile))decyl)) disulfide and that for the SAM on mercury was $\mathrm{HS}\left(\mathrm{CH}_{2}\right)_{n-1} \mathrm{CH}_{3}(n=14$, $16,18)$. The electrical properties of the junctions were characterized by current-voltage measurements. The ratio of the conductivity of the junction in the forward bias ( $\mathrm{Hg}$ cathodic) to that in the reverse bias $(\mathrm{Hg}$ anodic), at a potential of $1 \mathrm{~V}$, was $9 \pm 2$ when the SAM on mercury was derived from $\mathrm{HS}\left(\mathrm{CH}_{2}\right)_{15} \mathrm{CH}_{3}$. The ratio of the conductivity in the forward bias to that in the reverse bias increased with decreasing chain length of the alkanethiol used to form the SAM on mercury. These results demonstrate that a single redox center asymmetrically placed in a metal-insulator-metal junction can cause the rectification of current and indicate that a fixed dipole in the insulating region of a metal-insulator-metal junction is not required for rectification.
\end{abstract}

\section{Introduction}

We describe here a series of metal-insulator-metal (MIM) junctions that rectify current and contain a molecular electron acceptor. Molecular rectifiers-devices in which molecules allow the passage of a larger current in one bias than in the otherare potential components for memory and logic elements in molecular electronics. ${ }^{1-5}$ The structural requirements for molecular rectifiers are poorly understood. The most widely suggested and experimentally tested candidates for rectifiers are MIM junctions that contain donor-acceptor (D-A) compounds. ${ }^{1,2,5}$ The MIM junctions studied here do not contain a D-A pair but nonetheless rectify current; the observation that they are rectifiers suggests that neither an embedded molecular dipole nor a $\mathrm{D}-\mathrm{A}$ pair is essential for rectification. The data

* Corresponding authors. (G.M.W.) Telephone: (617)-495-9430. Fax: (617)-495-9857. E-mail: gwhitesides@gmwgroup.harvard.edu. (M.A.R.) Telephone: (0532)-291-162. Fax: (0532)-291-162. E-mail: rmp@unife.it.

$\dagger$ Harvard University.

University of Minnesota.

$\S$ Northwestern University.

" Universita' di Ferrara.

(1) Aviram, A. J. Am. Chem. Soc. 1988, 110, 5687-92.
(2) Metzger, R. M. Acc. Chem. Res. 1999, 32, 950-957.

(3) Joachim, C.; Gimzewski, J. K.; Aviram, A. Nature (London) 2000, 408 , $541-548$

(4) Ellenbogen, J. C.; Love, J. C. Proc. IEEE 2000, 88, 386-426.

(5) Aviram, A.; Ratner, M. A. Chem. Phys. Lett. 1974, 29, 277-83.

11730 - J. AM. CHEM. SOC. 2002, 124, 11730-11736 obtained in characterizing these junctions also provide a systematic view of the influence of the structure of the insulating layer containing the embedded electron acceptor on rectification in MIM junctions.

Molecular rectifiers were first suggested by Aviram and Ratner. ${ }^{5}$ Their design called for the placement of an organic molecule containing a covalently linked electron donoracceptor pair between two metal electrodes. The hypothesis underlying this design is based on the relative energies of the highest occupied molecular orbital (HOMO) of the donor unit and the lowest unoccupied molecular orbital (LUMO) of the acceptor: the current observed with one bias voltage (resulting from resonant electron transfer from the LUMO of the acceptor to the HOMO of the donor) would be greater that the current for the opposite bias (resulting from nonresonant transfer). ${ }^{5}$ Metzger and co-workers were the first to demonstrate experimentally a rectifier based on the design proposed by Ratner and Aviram; the rectifier was a MIM junction comprising a Langmuir-Blodgett ( $\mathrm{L}-\mathrm{B})$ film of $\gamma$-hexadecylquinolinium tricyanoquinomethanide supported on an aluminum electrode (with an $\mathrm{Al}_{2} \mathrm{O}_{3}$ surface layer) and an aluminum electrode formed by evaporation of metal onto the organic layer. ${ }^{2,6,7}$ The rectification in this system was attributed to the presence of the donor-acceptor molecule; recent work has shown that 
rectification is also observed if gold is used for both electrodes. ${ }^{8}$ These studies have clearly demonstrated rectification using donor-acceptor compounds, but the mechanism leading to the rectification is still the subject of discussion. ${ }^{9}$

Recent work has shown that rectifying MIM junctions can be fabricated using molecules that do not contain donoracceptor compounds. Heath et al. demonstrated a switchable MIM rectifier in which the insulating layer was composed of a Langmuir-Blodgett film of a rotaxane that has multiple redox states. ${ }^{10}$ A conformational change in the rotaxane molecule was suggested to be the origin of the switching behavior, but there are also other possibilities. Tour et al. fabricated a relatively simple rectifying junction based on a self-assembled monolayer (SAM) formed from 4-thioacetylbiphenyl on gold, with a second electrode of evaporated titanium. ${ }^{11}$ Rectification in this system was attributed to a Schottky barrier at the titanium electrode rather than some characteristic of the molecules in the SAM. ${ }^{11}$ These MIM junctions, and most other examples of moleculebased MIM junctions, share the feature that one electrode is formed by the evaporation of a metal film onto an organic monolayer. The interfacial structures that result from deposition of evaporated metal atoms onto organic films remain undefined, ${ }^{12}$ and their contribution, if any, to the electronic properties of MIM is not understood.

We have described a MIM junction having the structure metal/SAM//SAM/mercury in which the two metal-organic interfaces are formed under mild conditions (here "/" represents an interface with a covalent metal-organic bond and "//?" represents an interface between two SAMs in van der Waals contact). ${ }^{13-16}$ The junction is formed by mechanical contact between a SAM on a mercury drop and a SAM on silver or gold film supported by a silicon wafer (Figure 1). We are developing these junctions as a test bed for molecular electronics. For example, the system easily yields information about the electrical breakdown fields of $\mathrm{SAMs}^{13}$ and the distance dependence of tunneling through aliphatic and aromatic SAMs. ${ }^{15,16}$ We, ${ }^{14}$ Majda, ${ }^{17,18}$ and others ${ }^{19,20}$ have also examined related junctions in which the organic film is sandwiched between two

(6) Martin, A. S.; Sambles, J. R.; Ashwell, G. J. Phys. Rev. Lett. 1993, 70 $218-221$.

(7) A number of workers have demonstrated rectification by organic monolayers using scanning probe microscopy. We focus here on results obtained from measurements on ensembles of molecules. For example, see: Pomerantz, M.; A. Aviram; McCorkle, R. A.; Li, L.; Schrott, A. G. Science 1992 255, 1115-1118; Dhirani, A.; Lin, P.-H.; Guyot-Sionnest, P.; Zehner, R. W.; Sita, L. R. J. Chem. Phys. 1997, 106, 5249-5253.

(8) Xu, T.; Peterson, I. R.; Lakshmikantham, M. V.; Metzger, R. M. Angew. Chem., Int. Ed. 2001, 40, 1749-1752.

(9) Vuillaume, D.; Chen, B.; Metzger, R. M. Langmuir 1999, 15, 4011-4017.

(10) Wong, E. W.; Collier, C. P.; Behloradsky, M.; Raymo, F. M.; Stoddart, J. F.; Heath, J. R. J. Am. Chem. Soc. 2000, 122, 5831-5840.

(11) Zhou, C.; Deshpande, M. R.; Reed, M. A.; Jones L., II; Tour, J. M. Appl. Phys. Lett. 1997, 71, 611-613.

(12) Konstantine, K.; Zhang, P.; Opila, R. L.; Allara, D. L. Surf. Sci. 1995 338, 300-312.

(13) Haag, R.; Rampi, M. A.; Holmlin, R. E.; Whitesides, G. M. J. Am. Chem. Soc. 1999, 121, 7895-7906.

(14) Rampi, M. A.; Schueller, O. J. A.; Whitesides, G. M. Appl. Phys. Lett. 1998, 72, 1781-1783

(15) Holmlin, R. E.; Haag, R.; Chabinyc, M. L.; Ismagilov, R. F.; Cohen, A E.; Terfort, A.; Rampi, M.; Whitesides, G. M. J. Am. Chem. Soc. 2001 $123,5075-5085$

(16) Holmlin, R. E.; Ismagilov, R. F.; Haag, R.; Mujica, V.; Ratner, M. A.; Rampi, M.; Whitesides, G. M. Angew. Chem., Int. Ed. 2001, 40, 23162320 .

(17) Slowinski, K.; Fong, H. K. Y.; Majda, M. J. Am. Chem. Soc. 1999, 121, 7257-7261.

(18) Slowinski, K.; Majda, M. J. Electroanal. Chem. 2000, 491, 139-147.

(19) Porter, J. D.; Zinn-Warner, A. S. Phys. Rev. Lett. 1994, 73, 2879-82.

(20) Porter, J. D.; Zinn, A. S. J. Phys. Chem. 1993, 97, 1190-203.

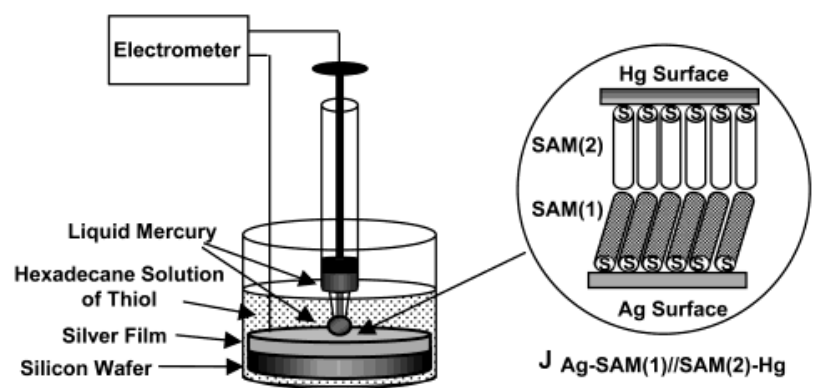

Figure 1. Schematic illustration of the fabrication of the MIM junction.

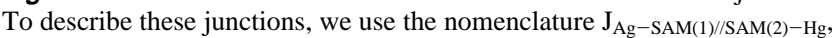
where $\mathrm{J}$ indicates a junction, $\mathrm{SAM}(1)$ indicates the SAM on the solid $\mathrm{Ag}$ electrode, and $\mathrm{SAM}(2)$ indicates the SAM on the $\mathrm{Hg}$ electrode. In these experiments, $\operatorname{SAM}(1)$ is formed from 1 and $\operatorname{SAM}(2)$ is formed from $\mathrm{HS}\left(\mathrm{CH}_{2}\right)_{n-1} \mathrm{CH}_{3}(n=14,16,18)$. We refer to SAMs composed of alkanethiolates with the composition $\mathrm{S}\left(\mathrm{CH}_{2}\right)_{n-1} \mathrm{CH}_{3}$ by the notation $\mathrm{C}_{n}$. The electrical junction is formed by mechanical contact of the two SAMs in a $1-10 \mathrm{mM}$ solution of the alkanethiol in hexadecane (the same solution used to form the SAM on the mercury drop). Details of the fabrication of the junction are provided in the Experimental Section.

mercury drops. We have preferred to study junctions with one solid and one liquid electrode rather than those with two liquid electrodes because they are more stable mechanically and because they do not show a compression phenomenon (characterized by Majda $)^{18}$ that leads to an upward drift in current at a fixed voltage over time. ${ }^{21}$

This paper describes measurements of the current-voltage $(I-V)$ characteristics of junctions consisting of a SAM formed from a compound containing tetracyanoquinodimethane (TCNQ) covalently linked to an alkanedisulfide (1) on silver or gold and<smiles>N#CC(C#N)=c1ccc(=C(C#N)C#N)c(CCCCCCCCCCSSCCCCCCCCCCc2cc(=C(C#N)C#N)ccc2=C(C#N)C#N)c1</smiles>

$\left(\mathrm{TCNQC}_{10} \mathrm{~S}\right)_{2}(\mathbf{1})$

a SAM formed from an alkanethiol on mercury. These junctions all rectify current.

The junctions represent a particularly useful model system for studying electrical rectification by organic thin films for the following reasons: (i) they do not contain a donor-acceptor compound or an embedded molecular dipole, (ii) neither electrode is formed by a process involving evaporation of metal atoms onto organic molecules, and (iii) the thickness of SAM on the mercury electrode can be varied systematically without extensive synthetic effort. They are better defined structurally than other model systems, although many aspects of the system - the metal-sulfur interface, the detailed structure of the SAM formed from (1) (which is disordered due to the disparity between the size of the TCNQ group and the methylene chain), and defects in the SAMs on the solid electrode and the mercury electrode-remain to be defined.

\section{Results and Discussion}

Junctions Incorporating 1 Rectify Current. The $\mathrm{J}_{\mathrm{Ag}-\mathbf{1} /(\mathrm{C} 16)-\mathrm{Hg}}$ junction rectifies current in the forward direction (positively

(21) The interfacial structure between the SAM on mercury electrode and the SAM on the solid electrode is difficult to define. While defects are certainly present in the SAMs and the solid electrode is not atomically smooth, the data from these junctions are reproducible and the trends are interpretable. 


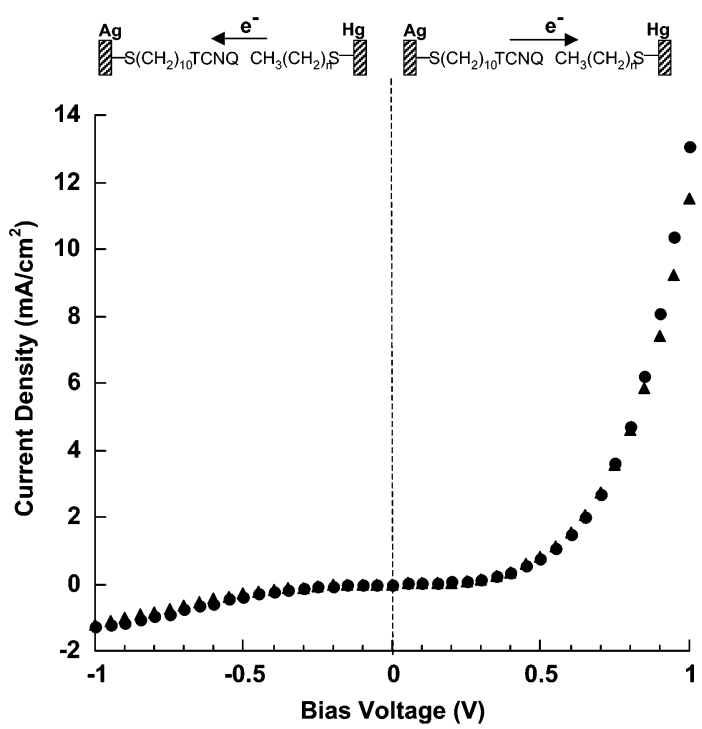

Figure 2. Current density against bias voltage for the $\mathrm{J}_{\mathrm{Ag}-1 / / \mathrm{C} 16-\mathrm{Hg}}$, junction. The plot shows two $I-V$ curves for the same junction (positively biased mercury electrode). The data were obtained by sweeping the applied voltage from 0 to $1 \mathrm{~V}$, from +1 to $-1 \mathrm{~V}$, and then from -1 to $0 \mathrm{~V}(\mathbf{})$; the entire sweep was repeated a second time $(\bullet)$ before disassembling the junction.

biased mercury electrode) relative to that the reverse direction (negatively biased mercury electrode) (Figure 2). The ratio of the forward to reverse bias current, $\mathrm{R}=|I(\mathrm{~V}) / I(-\mathrm{V})|$, is $9.0 \pm$ 2.0 at $1 \mathrm{~V}$ magnitude bias. This value of $R$ is close to the value obtained by Metzger (the average value of $R$ based on multiple junctions was 7.55 and the maximum for a particular junction was 27.5 at $2.2 \mathrm{~V})^{9,22}$ for MIM junctions comprising an insulating layer containing $\gamma$-hexadecylquinolinium tricyanoquinomethanide (HQ-TCNQ) and two gold electrodes. Our value of $R$ is $\sim 10$ times smaller than that reported by Ashwell $(R=$ 70 at $1 \mathrm{~V})^{23}$ for MIM junctions comprising $100 \mathrm{~L}-\mathrm{B}$ layers of hemicyanine and two gold electrodes. The current density and the value of $R$, at a given voltage, are nearly indistinguishable for $\mathrm{J}_{\mathrm{M}-1 / /(\mathrm{C} 16)-\mathrm{Hg}}$ junctions with either a $\mathrm{Ag}$ electrode or a $\mathrm{Au}$ electrode. The $I-V$ curves for these junctions are nearly indistinguishable over at least two potential cycles $(-1$ to +1 V). The mechanical stability of our configuration limits the number of potential cycles that we can routinely obtain for a given junction to approximately five; the most common failure mechanism is detachment of the mercury drop from the column of mercury in the syringe.

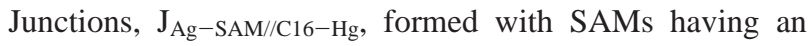
identical alkyl chain length and different functional groups, such as $\mathrm{HS}\left(\mathrm{CH}_{2}\right)_{10} \mathrm{COOH}$ and $\mathrm{HS}\left(\mathrm{CH}_{2}\right)_{10} \mathrm{CO}-\eta-\left(\mathrm{C}_{5} \mathrm{H}_{5}\right)_{2} \mathrm{Fe}$, do not rectify. The current densities observed in the junction $\mathrm{J}_{\mathrm{Ag}-1 / /(\mathrm{C} 16)-\mathrm{Hg}}$ are $\sim 10^{3}$ higher than those for junctions where the SAM on Ag was formed from thiols with smaller headgroups but equal alkyl chain lengths (for example, $\mathrm{HS}\left(\mathrm{CH}_{2}\right)_{10} \mathrm{COOH}, \mathrm{HS}\left(\mathrm{CH}_{2}\right)_{10^{-}}$ $\mathrm{CH}_{3}$ ). Possible explanations for the larger current density include the following: (i) The junction contains regions that are thinner than the average thickness $(1.2 \mathrm{~nm})$ due to disorder in the SAM

(22) Metzger, R. M.; Xu, T.; Peterson, I. R. J. Phys. Chem. B 2001, 105, 72807290 .

(23) Ashwell, G. J.; Gandolfo, D. S. J. Mater. Chem. 2001, 11, 246-248. Ashwell and co-workers have observed rectification using different donor acceptor systems in a MIM jumnction: Ashwell, G. J.; Gandolfo, D. S. J. Mater. Chem. 2002, 12, 411-415. Ashwell, G. J.; Gandolfo, D. S.; Hamilton, R. J. Mater. Chem. 2002, 12, 416-419. formed from $1 .^{24}$ (ii) Mechanical compression of the SAMs under potential bias decreases the thickness of the insulating layer. $^{25}$ (iii) The presence of TCNQ facilitates electron transport in the junction relative to a SAM of similar thickness.

Several spectroscopic data lead us to believe that disorder in the SAM formed from $\mathbf{1}$ is at least one significant contributor to the larger current density. The thickness of the monolayer formed by 1 on gold determined by ellipsometry is $1.2 \mathrm{~nm}$, a value significantly smaller than the value of $\sim 1.8 \mathrm{~nm}$ estimated by the assumption of trans-extended molecules at a tilt of $\sim 30^{\circ}$ with respect to the surface normal. ${ }^{26}$ Polarized infrared external reflectance spectra (PIERS) of the SAM of 1 on gold contained methylene stretches at 2928 and $2857 \mathrm{~cm}^{-1}$, values that indicate a disordered monolayer. ${ }^{24,27}$ The surface coverage of TCNQ groups determined by integration of the waves in a cyclic voltammogram is $\sim 1.4$ molecule $/ \mathrm{nm}^{2}$. This coverage is smaller than that $\left(2.1 \mathrm{molecule} / \mathrm{nm}^{2}\right)$ for alkanethiolate monolayers, an observation that is not surprising considering that the width of the TCNQ headgroup ( $\sim 9 \AA$ ) is nearly twice the lattice spacing ( $\sim 5 \AA$ ) observed for SAMs of alkanethiols on gold and silver(111). Although these data indicate that the monolayer is disordered, the $I-V$ measurements are reproducible from junction to junction. This reproducibility suggests that we are measuring an average property of the molecules composing the SAM.

Rectification Depends on the Fractional Coverage of TCNQ. To determine the effects of the structural order of the SAM of 1 and the dependence of the current density and $R$ on the coverage of TCNQ groups, we formed mixed monolayers from solutions at 1:10, 1:100, and 1:1000 mole fractions of $\mathbf{1}$ and decyl disulfide, $\left(\mathrm{CH}_{3} \mathrm{C}_{9} \mathrm{H}_{2} \mathrm{~S}\right)_{2}$, at $\sim 1 \mathrm{mM}$ total disulfide concentration. As the mole fraction of $\mathbf{1}$ in the solution used to form the SAMs decreases, the SAMs show increasing ordering (determined by the position of the methylene peaks in infrared spectra) and decreasing concentration of TCNQ (determined electrochemically from peak areas in CVs). The CVs showed that the surface coverage of TCNQ in the SAM decreased by the same magnitude as the mole fraction of $\mathbf{1}$ in solution relative to the SAM formed from a solution of $\mathbf{1}$ alone.

The absolute current densities decreased with decreasing mole fraction of $\mathbf{1}$ used to form the monolayer: for example, the current density at $1 \mathrm{~V}$ for the SAM from the 1:100 mixture of 1 and decyl disulfide $\left(\mathrm{C}_{10}\right)_{2}$ is $\sim 50$ times smaller than that for SAM formed from pure 1. Junctions formed with mixed SAMs incorporating $\mathbf{1}$ and $\left(\mathrm{C}_{10}\right)_{2}$ also rectified current, and the value of the rectification ratio decreased with decreasing concentration of $\mathbf{1}$. For a SAM formed from a solution of $[\mathbf{1}]:\left[\left(\mathrm{C}_{10}\right)_{2}\right]=1: 100$, $R$ was $\sim 4$ compared to $\sim 10$ for the pure SAM of 1. Despite the relatively large decrease in number of TCNQ moieties present in the mixed SAM, the junctions have a relatively small change in $R$ (a factor of $\sim 2$ ).

(24) Skulason, H.; Frisbie, C. D. Langmuir 1998, 14, 5834-5840.

(25) Majda and co-workers have observed this behavior in a similar MIM junction where both metal electrodes are formed from liquid mercury. Ref $17,18$.

(26) The refractive index of the SAM was assumed to be the same as that for a SAM of an alkanethiolate (1.45). We do not believe that the assumption of this value of the refractive index will create a substantial error in the derived value of the thickness.

(27) Porter, M. D.; Bright, T. B.; Allara, D. L.; Chidsey, C. E. D. J. Am. Chem. Soc. 1987, 109, 3559-3568. 


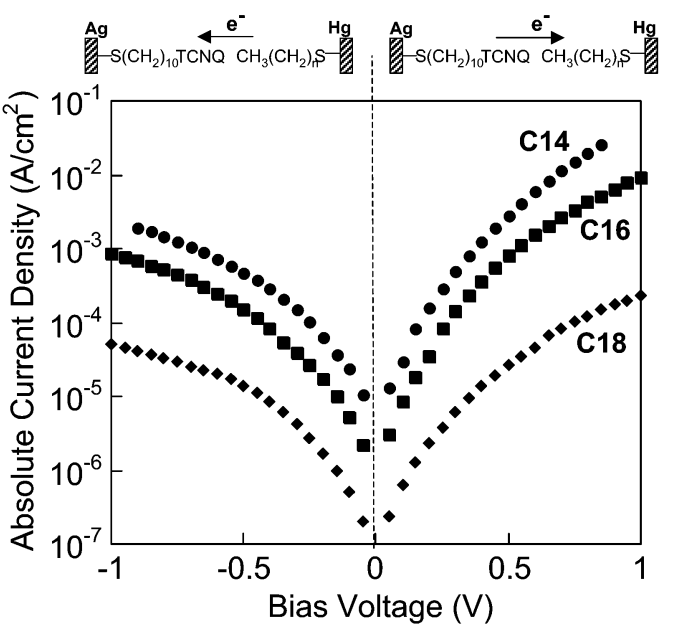

Figure 3. Current density against bias voltage for the $\mathrm{J}_{\mathrm{Ag}-1 / / \mathrm{C}_{\mathrm{n}}-\mathrm{Hg}}$ junctions, where $n=14,16,18$.

These results can be understood if the molecules in the SAM on the silver electrode conduct current independently of each other. That is, the data are compatible with the hypothesis that rectification is a single-molecule property rather than a property of aggregates of TCNQ groups. The current density measured for junctions with a single-component SAM of $\mathbf{1}$ is $\sim 1000$ times higher than that for junctions with a single-component SAM formed from decyl disulfide. If the conductance of the mixed SAMs is linearly related to their composition and the molecules conduct independently, then the conductance of the molecules of 1 should dominate the total conductance of the junction until the composition of the SAM is approximately $[\mathbf{1}]:\left[\left(\mathrm{C}_{10}\right)_{2}\right]=$ $1: 1000$. Both the data for the current density through the junction and for $R$ qualitatively agree with this interpretation; relatively large changes in the coverage of TCNQ are necessary to cause a decrease in $R$. Unfortunately, these results are not sufficient to allow us to separate unambiguously the contribution of the TCNQ groups and the structural order of the monolayer to the absolute magnitude of the current density. We expect the structural order of the monolayer to increase with increasing composition of the alkylthiolate, but the concentration of TCNQ groups obviously decreases concomitantly. Thus, the data cannot disentangle both effects. They do indicate that the rectification ratio depends on the mole fraction of TCNQ groups in the SAM but do not specify the form of the dependence.

Rectification Depends on the Thickness of the SAM on Mercury. We have also studied junctions where we varied the SAM on the mercury electrode. Figure 3 shows the currentvoltage $(I-V)$ curves for the junctions, $\mathrm{J}_{\mathrm{Ag}-\mathbf{1} / / \mathrm{C}_{\mathrm{n}}-\mathrm{Hg}}(n=14$, $16,18)$. Over this limited range of lengths, the magnitude of the current decays exponentially $\left(I \propto \mathrm{e}^{-\beta d}\right.$, where $d$ is the tunneling distance and $\beta=\sim 1.0 \AA^{-1}$ ) at a fixed bias in the forward direction with increasing chain lengths of the alkanethiol used to from the SAM on $\mathrm{Hg}$. This value is in the range observed in a variety of measurements of the distance dependence of tunneling in alkane oligomers $\left(\sim 0.9-1.4 \AA^{-1}\right) .15,17,28$ All of the junctions, $\mathrm{J}_{\mathrm{Ag}-1 / / \mathrm{C}_{\mathrm{n}}-\mathrm{Hg}}(n=14,16,18)$, rectify (Figure 3 ). For $\mathrm{C}_{16}$ and $\mathrm{C}_{18}$, the ratio of the current at $+1 \mathrm{~V}$ to that at $-1 \mathrm{~V}$ decreases with increasing alkyl chain length of the SAM on $\mathrm{Hg} ; R=9.0 \pm 2.0$ for $\mathrm{C}_{16}$ and $R=4.0 \pm 0.8$ for $\mathrm{C}_{18}$. We

(28) Smalley, J. F.; Feldberg, S. W.; Chidsey, C. E. D.; Linford, M. R.; Newton, M. D.; Liu, Y.-P. J. Phys. Chem. 1995, 99, 13141-13149. are unable to reach $\pm 1 \mathrm{~V}$ with the junction containing $\mathrm{C}_{14}$ because this junction broke down electrically at $\pm 0.85 \mathrm{~V}$. At the highest bias attainable $(0.85 \mathrm{~V})$, however, $R=15 \pm 3$ for $\mathrm{C}_{12}$; this number can be compared with that for $\mathrm{C}_{16}$ at this voltage, $R=7.0 \pm 1.4$. Thus, within the limited range of compositions over which we have data, each additional $\mathrm{CH}_{2}$ group decreases $R$ by a factor of $\sim 2$. These results demonstrate that both the conductivity and the ability of the junction to rectify are affected by the chain length of the SAM on the mercury electrode.

Origin of Molecular Rectification. Why do junctions incorporating TCNQ groups rectify? We can eliminate several possible causes, but we cannot identify a simple mechanism unambiguously. Metal oxide formation is not the cause of the rectification because neither gold nor silver forms a surface oxide when coated by a SAM. ${ }^{29}$ The rectification does not appear to be due to the difference in work functions between the metals in the junction. We observe no change in $R$ on changing the electrode to gold from silver, even though the work function of gold is $5.3 \mathrm{eV}$ and that of silver is $4.5 \mathrm{eV}$ (nearly indistinguishable from that of mercury, $4.5 \mathrm{eV}) .{ }^{30}$ Disorder in the SAM of $\mathbf{1}$ is unlikely to be the sole cause, since the junction formed with a SAM from a thiol with headgroup similar in size to TCNQ-ferrocene-does not rectify. These results suggest that the rectification is caused by a molecular property of the molecules that bridge the metal electrodes.

The molecular bridge in all the studied junctions, junctions $\mathrm{J}_{\mathrm{Ag}-\mathbf{1} / /(\mathrm{C} 16)-\mathrm{Hg}}$ and junctions, $\mathrm{J}_{\mathrm{Ag}-\mathrm{SAM} / / \mathrm{C} 16-\mathrm{Hg}}$, where the SAMs have an identical alkyl chain length and different functional groups, (e.g., $\mathrm{HS}\left(\mathrm{CH}_{2}\right)_{10} \mathrm{COOH}$ and $\mathrm{HS}\left(\mathrm{CH}_{2}\right)_{10} \mathrm{CO}-\eta-\left(\mathrm{C}_{5} \mathrm{H}_{5}\right)_{2}-$ $\mathrm{Fe}$ ), are compositionally heterogeneous to the extent that they contain two types of chemical functional groups - a series of methylene units and different functional groups (TCNQ, COOH, $\left.\left(\mathrm{C}_{5} \mathrm{H}_{5}\right)_{2} \mathrm{Fe}\right)$. This heterogeneity causes these junctions to be asymmetric in several regards: (i) the electronic structure of the molecular units that bridge the two electrodes is inhomogeneous; the local electronic energy levels of the functional groups are clearly distinct from those of methylene groups; ${ }^{31,32}$ (ii) the functional groups are asymmetrically coupled electronically to the two electrodes; they are covalently linked to the Ag electrode through an alkane chain and they are in van der Waals contact with the SAM on the Hg electrode; and (iii) the functional groups are asymmetrically placed spatially; in all cases, the thickness of the SAM on mercury is greater than that

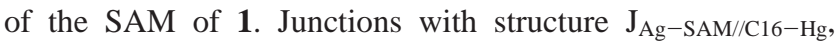
formed with SAMs having the same chain length and different terminal functional groups (e.g., $\mathrm{HS}\left(\mathrm{CH}_{2}\right)_{10} \mathrm{COOH}$ and $\mathrm{HS}$ $\left.\left(\mathrm{CH}_{2}\right)_{10} \mathrm{CO}-\eta-\left(\mathrm{C}_{5} \mathrm{H}_{5}\right)_{2} \mathrm{Fe}\right)$, do not rectify. The dissimilarity of the molecular structure of the SAMs on the two electrodes alone does not result in rectification.

(29) Metzger has shown that the rectification in MIM junctions formed with HQ-TCNQ is not caused by electrodes containing a metal oxide as well: $\mathrm{Xu}, \mathrm{T}$.; Peterson, I. R.; Lakshmikantham, M. V.; Metzger, R. M. Angew. Chem., Int. Ed. 2001, 40, 1749-1752.

(30) Weast, R. C., Ed. CRC Handbook of Chemistry and Physics; CRC Press: Boca Raton, FL, 1987.

(31) We base our discussion on a valence orbital representation and treat the orbitals of TCNQ separately from those of the alkyl chain. The $\pi$ symmetry states of TCNQ are unlikely to mix strongly with the alkyl chain due to symmetry and difference in energy.

(32) Bartmess, J. E. In NIST Chemistry WebBook, NIST Standard Reference Database 69; Mallard, W. G., Linstrom, P. J., Eds.; National Institute of Standards and Technology: Gaithersburg, MD, 1998. 
A

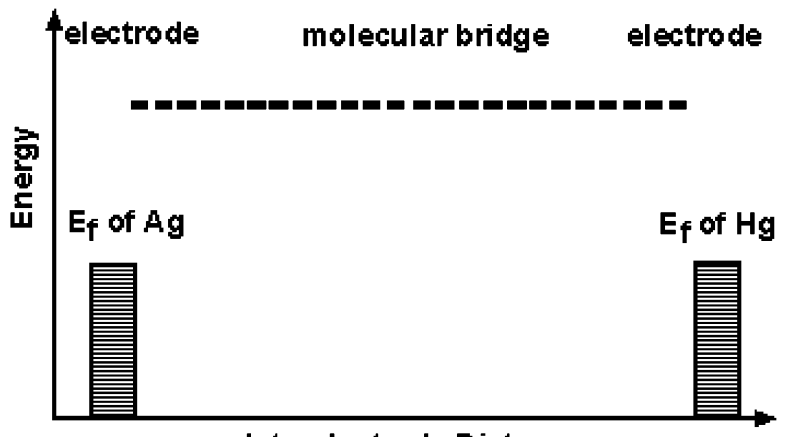

Interelectrode Distance

B

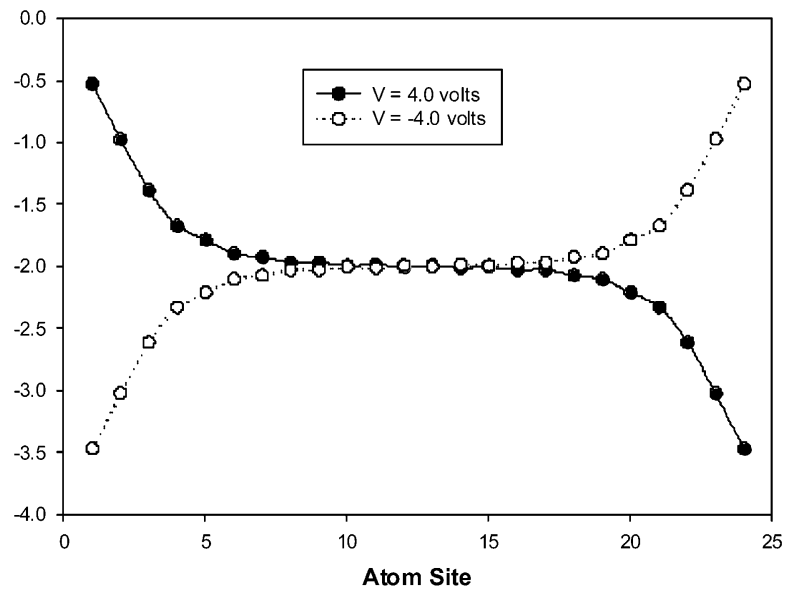

C

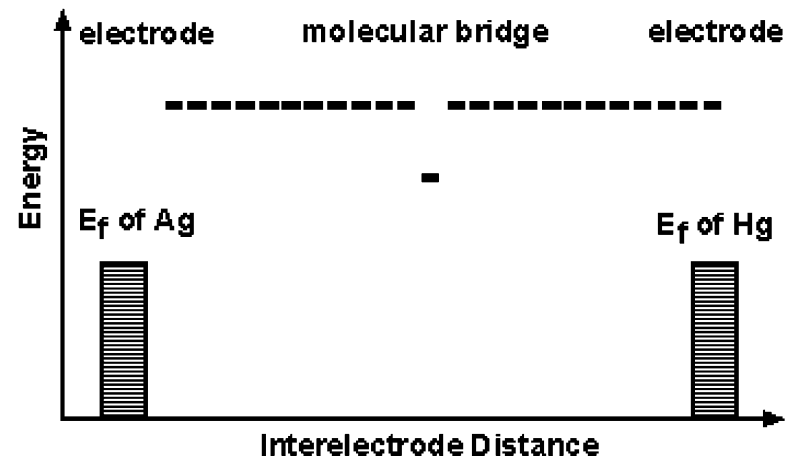

D

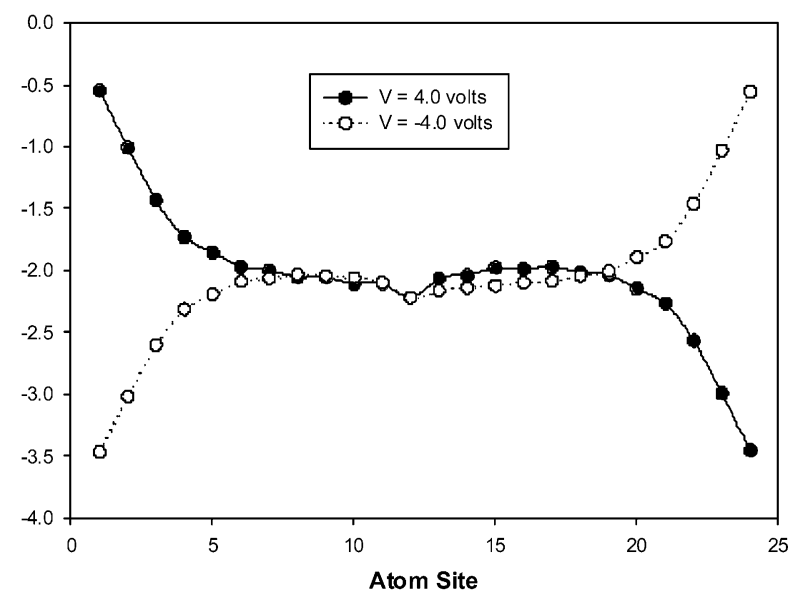

Figure 4. Schematic illustration of the profile of the electrostatic potential with position in a molecular MIM junction based on a tight binding model. ${ }^{34}$ (A) Energy level diagram for a homogeneous molecular bridge between two electrodes. In the homogeneous case, all of the sites in the molecular bridge have the same energy relative to the Fermi levels of the electrodes and have equal site-coupling energies. This case ignores all electronic effects due to van der Waals interaction. (B) Diagram of electrostatic potential for the homogeneous system in (A) under potential bias (solid and dashed lines represent opposite biases; 20 atoms connected to two metallic atoms on each side). If a potential bias is applied to a homogeneous junction, most of the potential drop occurs at the metal-molecule interfaces, and there is little or no drop through the molecular bridge. The potential is symmetric with respect to the electrical bias. (C) Energy level diagram for a molecular bridge with one inhomogeneous site between two electrodes. In the simplest heterogeneous case, one site has a different site energy than does the rest of the molecular bridge and has a different site-coupling energy to its neighbors. The site coupling represents the van der Waals interface. (D) Diagram of electrostatic potential for the heterogeneous system in (C) under potential bias (solid and dashed lines represent opposite biases; 20 atoms connected to two metallic atoms on each side, a defect at atomic site 12, and a weak link between atoms 12 and 13). If a potential is applied to the junction, a drop in potential can occur through a site that has a substantially different site energy or site coupling. The potential is asymmetric with respect to bias in this case; this asymmetry will result in rectification. ${ }^{38}$

The electrical properties of the $\mathrm{J}_{\mathrm{Ag}-\mathbf{1} / /(\mathrm{C} 16)-\mathrm{Hg}}$ junctions can be described by a tight binding model that includes, in addition, a simultaneous self-consistent solution of the Poisson equation. ${ }^{33-36}$ In this theoretical framework, the molecular bridge that connects the two electrodes is decomposed into sites that have an associated energy relative to the Fermi levels of the electrodes (Figure $4 \mathrm{~A}$ and $\mathrm{C}$ ) and a site coupling that describes the quantum-mechanical interaction between a site and its neighbors. ${ }^{37}$

A complete theoretical analysis of rectification is complex and will be described elsewhere ${ }^{38}$ but we can address the most

(33) Datta, S.; Tian, W.; Hong, S.; Reifenberger, R.; Henderson, J. I.; Kubiak, C. P. Phys. Rev. Lett. 1997, 79, 2530-2533.

(34) Mujica, V.; Roitberg, A. E.; Ratner, M. J. Chem. Phys. 2000, 112, 68346839

(35) Mujica, V.; Ratner, M. A. Chem. Phys. 2001, 264, 365-370.

(36) Ratner, M. A. J. Phys. Chem. 1990, 94, 4877-4883.

(37) Previous work has shown that a simple tight-binding model is adequate to describe electron transport in junctions where the molecules that bridge the electrodes are nearly structurally homogeneous (e.g., junctions such as $J_{\mathrm{Ag}-(\mathrm{Cn}) / /(\mathrm{C} 16)-\mathrm{Hg}}$ where the insulating layer contains only one type of functional group). See ref 16.

(38) Gonzalez, C.; Mujica, V.; Ratner, M. Ann. New York Acad. Sci. 2002, 960 161-176. Mujica, V.; Nitzan, A.; Ratner, M. A. Chem. Phys. 2002, 281, $147-150$ important experimental findings through a qualitative analysis of the system. Equation 1 represents a standard elastic scattering

$$
\begin{aligned}
& I(V)=\frac{2 e}{h} \Delta_{0}^{2} \int_{E_{\mathrm{f}}-e V}^{E_{\mathrm{f}}}\left|G_{1 N}(\eta, V)\right|^{2} \mathrm{~d} \eta \\
& G_{1 N}^{-1}(\eta)=\left\langle u_{1}\left|H-\eta-\sum(\eta)\right| u_{N}\right\rangle
\end{aligned}
$$

formulation of the current-voltage dependence of a molecular MIM junction ( $e$ is the charge of an electron, $h$ the Planck's constant, $V$ the applied voltage, $E_{\mathrm{f}}$ the Fermi energy of the electrode, $\Delta_{0}$ the spectral density of the electrodes, $G$ the element of the Green's function for the system as defined in eq 2, and $\eta$ the energy variable). ${ }^{39-42}$ In this expression, the molecular bridge is assumed to interact with the electrodes through a single

(39) Ratner, M. A.; Davis, B.; Kemp, M.; Mujica, V.; Roitberg, A.; Yaliraki, S. Ann. N. Y. Acad. Sci. 1998, 852, 22-37.

(40) Tian, W.; Datta, S.; Hong, S.; Reifenberger, R.; Henderson, J. I.; Kubiak, C. P. J. Chem. Phys. 1998, 109, 2874-2882.

(41) Samanta, M. P.; Tian, W.; Datta, S.; Henderson, J. I.; Kubiak, C. P. Phys Rev. B: Condens. Matter 1996, 53, R7626-R7629.

(42) Hall, L. E.; Reimers, J. R.; Hush, N. S.; Silverbrook, K. J. Chem. Phys. 2000, 112, 1510-1521 
surface atomic orbital on the sulfur atom that is chemically bonded to the electrode. The Green function for the system contains the molecular Hamiltonian for the system and is given in eq 2 ( $u$ is the interfacing orbitals of sulfur on the left, 1 , and the right, $N$, of the molecular bridge, $H$ is the molecular Hamiltonian, $\eta$ is the energy variable, and $\sum$ is the full selfenergy term for the electrodes). We assume here that the Fermi levels $E_{\mathrm{F}}$ of the electrodes are nearly the same, as is true for $\mathrm{Hg}$ and $\mathrm{Ag}$, that the spectral density of the electrodes is independent of voltage and that we can ignore any temperature effects. The model also assumes that the electron is not localized on any portion of the molecular bridge for an appreciable time during the electron transport process; that is, the mechanism of electron transport does not involve a hopping step to a trap state. $^{43}$

To understand the requirements for the rectification of current, we can examine the symmetry of the element of the Green function (eq 2) in eq 1 with respect to voltage. The molecular bridge-electrode coupling (the self-energy of the metal contact) is voltage-dependent and is a symmetric function of $V$; that is, $\Sigma(\mathrm{V})=\Sigma(-\mathrm{V})$. The source of an asymmetric dependence of current on voltage must be in the Hamiltonian that describes the electronic structure of the molecular bridge. The Hamiltonian can depend on voltage if there is a potential drop through the molecular bridge (See Figure 4). ${ }^{44}$ The potential drop can be asymmetric with respect to voltage as a result of an electronic or structural asymmetry in the molecule. In this event, the molecular Hamiltonian, and therefore the Green function, will not be symmetric under bias inversion. This asymmetry will lead the rectification ratio, $R$, to be different from unity. Vuillaume et al. also recently suggested that an asymmetry in the shape of the electrostatic potential is a contributing factor in rectification by $\mathrm{D}-\mathrm{A}$ molecules in MIM junctions. ${ }^{45}$

The observation of rectification in the junctions studied here can be understood using this simple model. We have calculated current-voltage curves for the MIM junction from a selfconsistent calculation of the Schrödinger and the Poisson equations. ${ }^{34}$ Our first calculations were performed with a model where the molecular bridge contained one inhomogeneous site with a lower site energy than the rest of the bridge, but with equal site couplings. Because of the assumption of equal-site coupling energies, these calculations ignore the electronic effects of the van der Waals contact. Our calculations indicate that in this case (i) rectification will occur, (ii) $R$ will increase on increasing the energy difference between the inhomogeneous site and the rest of the bridge, and (iii) $R$, even for TCNQ, a very low energy site, will be relatively small $(<2)$. Significantly larger rectification ratios are observed experimentally in the

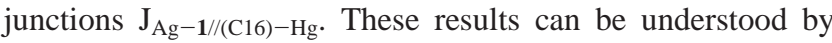
inclusion of both the electronic effects of the lower local site energy near TCNQ and the non-bonded contact between the two SAMs in the junction. In the tight binding model, the van

(43) It is difficult to determine whether this assumption is valid for the junctions studied here. We assume that the qualitative predictions of the model are valid, but a more detailed analysis of the system will require testing of this assumption.

(44) Experimental evidence and model theoretical calculations indicate that, for many homogeneous molecular MIM junctions, the voltage drops at the two metal-molecule interfaces and also across the molecular bridge: Samanta, M. P.; Tian, W.; Datta, S.; Henderson, J. I.; Kubiak, C. P. Phys. Rev. B 1996, 53, R7626-R7629. Damle, P. S.; Ghosh, A. W.; Datta, S. Phys. Rev. B 2001, 64, 201403-1.

(45) Krzeminski, C.; Delerue, C.; Allan, G.; Vuillaume, D.; Metzger, R. M. Phys. Rev. B 2001, 64, 085405-1. der Waals interaction can be modeled by weakening one of the site couplings to the TCNQ group. Initial calculations indicate that the weak electronic link and a low local site energy can cause a relatively large voltage drop near the TCNQ moiety and lead to a significant rectification ratio $(>10)$.

The dependence of the rectification ratio $R$ on the length of the SAM on mercury is an unexpected feature of our measurements. Simple tight-binding calculations show that $R$ will behave in a nonmonotonic fashion as a function of the length of the molecular bridge for any choice of the electronic coupling at the weak link and inhomogeneous site energy. ${ }^{38}$ This result may be due, in part, to local orbital degeneracy. For example, if the energy levels of the two SAMs that compose the molecular bridge are nearly degenerate, they will mix strongly. In this case, the value of $R$ may change as the local energy levels are brought closer or farther from resonance, due to variations in the alkyl chain length.

These initial calculations indicate that the actual behavior of $R$ as a function of the length of the alkyl chains in the SAM should be system-dependent. It will, therefore, be difficult to make generalizations about the specific behavior of the currentvoltage relationships in MIM junctions with heterogeneous molecular bridges. This difficulty is simply a result of the number of variables - the length of the molecular bridge, the site energies within the molecular bridge, and the applied voltage - that contribute to the rectification. Further calculations are in progress on this issue.

\section{Conclusions}

We have demonstrated a metal/SAM//SAM/mercury junction that rectifies current. This junction incorporates a chemical functionality (TCNQ) with low-lying vacant molecular orbital; we infer that the presence of this TCNQ group is the cause of rectification. We also observed that the rectification ratio at a given voltage decreases with increasing thickness of the SAM on the mercury electrode. Our results demonstrate that rectification can be achieved in a metal-insulator-metal junction if an electron acceptor is placed in the insulating layer. These results do not discount the previous model for rectification using a donor-acceptor pair but do suggest that rectification can be obtained with only an electron acceptor. ${ }^{5}$ The detailed mechanism of electron transport is still under investigation, but a simple tight-binding model suggests that the presence of a single inhomogeneous functional group, with energy significantly different from the rest of the sites of the molecular bridge, can lead to rectification in a MIM and that stronger rectification can be obtained if the bridge includes an electronic weak link such as a van der Waals interaction.

The ease of fabrication of the Ag-SAM//SAM-Hg junction makes it straightforward to investigate the influence of molecular structure on electron transport through organic thin films much more easily than with other approaches that are commonly used to study molecular electronics. These junctions have three advantages in the study of the structural requirements for molecular rectifiers. (i) The junction is easy to assemble and does not require extensive specialized equipment for fabrication. (ii) The molecular composition of the junction is modular; the SAM on the mercury electrode can be changed independently of the SAM on the solid electrode. (iii) The junction makes it possible to form and characterize statistically significant numbers 
of junctions in a short period of time (days). The disadvantages of the system include the inability to study rectification at a single (or few)-molecule level, the structural ambiguities at the interfaces at the electrodes and at the SAM-SAM interface, and the inability to easily use these junctions at cryogenic temperatures. We believe that the system will be a useful tool for the discovery of the microscopic chemical properties that control the macroscopic electrical properties of molecule-based electrical junctions.

\section{Experimental Section}

Materials. Alkanethiols ( $\left.\mathrm{HS}\left(\mathrm{CH}_{2}\right)_{n-1} \mathrm{CH}_{3}(n=14,16,18)\right)$ were purchased from Aldrich or TCI and were used without further purification. Electronic grade mercury $(99.9998 \%)$ was purchased from Alpha. Caution: Mercury is highly toxic if swallowed or if its vapors are inhaled. Hexadecane was purchased from Aldrich.

Formation of SAMs. The silver film was prepared by thermal evaporation (Edwards Auto 306) of an adhesion layer of chromium ( $\sim 60 \AA$ thick) onto a 3-in. $\mathrm{Si} / \mathrm{SiO}_{2}$ wafer (Silicon Sense, test grade) followed by a layer of silver ( $\sim 2000 \AA$ thick). SAMs on silver (or gold) films were formed by immersion of a freshly evaporated thin film in a 1-5 mM solution of bis(20-(2-((2,5-cyclohexadiene-1,4diylidene)dimalonitrile))decyl)) disulfide (1) in $\mathrm{CH}_{2} \mathrm{Cl}_{2}$ for $24 \mathrm{~h}$. The synthesis of $\mathbf{1}$ has been described previously. ${ }^{24}$ Mixed monolayers were formed by immersion of the silver substrate in mixtures of $\mathbf{1}$ and decyl disulfide in $\mathrm{CH}_{2} \mathrm{Cl}_{2}$. The SAMs were characterized by ellipsometry, infrared spectrometry, and X-ray photoelectron spectroscopy.

Fabrication of the Junctions. The junctions were assembled as described previously. ${ }^{13,15,16}$ We describe the procedure briefly here. A SAM-coated silver film was placed in a beaker and covered with a solution of hexadecane containing the thiol used to form the SAM on mercury (usually hexadecanethiol) at a concentration of $\sim 1-10 \mathrm{mM}$. Experiments were also performed at lower concentrations of alkanethiol, $<0.1 \mathrm{mM}$, to verify that the SAM on the silver film was stable with respect to any potential exchange reactions with the thiol in the hexadecane bath. The SAM on mercury was formed in situ on an $\sim 1$-mm-diameter drop suspended from a gastight syringe $(1 \mathrm{~mL}$; Hamilton). We allowed the SAM on $\mathrm{Hg}$ to form over 5-10 $\mathrm{min}$ in the
$10 \mathrm{mM}$ solution of alkanethiol $\left(\mathrm{HS}\left(\mathrm{CH}_{2}\right)_{n-1} \mathrm{CH}_{3}\right.$, where $n=14,16$, 18 ) in hexadecane. A micromanipulator allowed mechanical contact between the SAM on the $\mathrm{Hg}$ drop and the SAM on the metal film. We measured the area of contact with a CCD camera with a magnifying lens. Typical contact areas ranged from $4 \times 10^{-3}$ to $7 \times 10^{-3} \mathrm{~cm}^{2}$.

Current-Voltage Measurements. The $\mathrm{Ag}-\mathrm{SAM}$ was connected electrically to an electrometer (Keithley model 6517) by an alligator clip in contact with the silver surface. A tungsten wire protruding from the Teflon tip of the plunger in the syringe provided an electrical connection between the mercury electrode and an electrometer. The $I-V$ curves were measured by using the electrometer to provide the bias voltage and to measure the current. The potential was increased in steps over the range of 0 to $1 \mathrm{~V}$, and the current was read $4 \mathrm{~s}$ after each step in voltage.

Current-voltage $(I-V)$ measurements were obtained at both positive and negative biases at least two times for the same junction. Measurements were repeated for at least three spots on at least three wafers for each type of junction. All current measurements have an approximate error of $\pm 20 \%$. The largest contribution to uncertainties in separate trials is the uncertainty of the area of contact between the solid and liquid surfaces.

The maximum voltage that could be applied for the junctions was $\sim 1 \mathrm{~V}$ for the longer SAMs $(\geq 16)$ and $\sim 0.7 \mathrm{~V}$ for the shorter $(<14)$ SAMs on $\mathrm{Hg}$. At higher voltages, the current would increase dramatically and lead to electrical breakdown of the junction with further applied potential. Some junctions $(\sim 10 \%)$ exhibited electrical breakdown at low bias voltages (less that $0.2 \mathrm{~V}$ ). The cause of the instability is typically a small scratch on the metal wafer or contamination of the hexadecane solution by dust. The data from these junctions were not included in the analysis.

Acknowledgment. This work was supported by the ONR, DARPA, and the NSF (ECS-9729405). M.L.C. and R.E.H. thank the National Institutes of Health for postdoctoral fellowships. C.D.F. thanks the Center for Interfacial Engineering (an NSF ERC) at the University of Minnesota for funding.

JA020506C 